\title{
The Relationship Enfolded in Bohr's Quantum Condition and a Previously Unknown Formula for Kinetic Energy
}

\author{
Koshun Suto ${ }^{1}$ \\ ${ }^{1}$ Chudaiji Buddhist Temple, Isesaki, Japan \\ Correspondence: Koshun Suto, Chudaiji Buddhist Temple, 5-24, Oote-Town, Isesaki, 372-0048, Japan. E-mail: \\ koshun_suto129@mbr.nifty.com
}

Received: December 2, 2018

Accepted: December 20, 2018

Online Published: January 29, 2019

doi:10.5539/apr.v11n1p19

URL: https://doi.org/10.5539/apr.v11n1p19

\begin{abstract}
Bohr's quantum condition is an indispensable assumption for classical quantum theory. However, strictly speaking, Bohr's quantum condition does not hold when deriving the energy of an electron forming a hydrogen atom from the perspective of the theory of relativity. In this paper, it is thought that the relationship enfolded in Bohr's quantum condition, i.e., $v_{n} / c=\alpha / n$ is suitable as a new quantum condition to replace Bohr's quantum condition. Also, in quantum mechanics, the energy of an electron is derived based on the theory of relativity, as exemplified in the theory of Sommerfeld. However, this paper points out that the previous energy formula based on the theory of relativity is mistaken. It also proposes a previously unknown formula for the kinetic energy of an electron.
\end{abstract}

Keywords: Hydrogen Atom, Kinetic Energy, Bohr's Quantum Condition, Einstein's Energy-Momentum Relationship, Planck Constant

\section{Introduction}

N. Bohr was the first to derive the energy levels of an electron forming a hydrogen atom (this will be abbreviated below as energy levels of the hydrogen atom). This Introduction reviews the history up to derivation of the energy levels of the hydrogen atom with the assistance of the writings of Dr. H. Ezawa in Japanese.

In 1884, J. J. Balmer noticed that the wavelengths $\lambda$ of the spectral lines emitted from a hydrogen atom could be described with the following formula.

$$
\lambda=B \frac{n^{2}}{n^{2}-4}, \quad B=3.6456 \times 10^{-7} \mathrm{~m} .
$$

After that, W. Ritz transformed this formula as follows.

$$
\frac{1}{\lambda}=\frac{4}{B}\left(\frac{1}{2^{2}}-\frac{1}{n^{2}}\right), \quad n=3,4, \cdots .
$$

Ritz also generalized Equation (2) as follows.

$$
\frac{1}{\lambda}=R\left(\frac{1}{m^{2}}-\frac{1}{n^{2}}\right), \quad n=m+1, \quad m+2, \cdots ; m=1,2, \cdots .
$$

Bohr tried multiplying both sides of Equation (3) by $h c$. When this is done,

$$
h \frac{c}{\lambda}=h v=\frac{h c R}{m^{2}}-\frac{h c R}{n^{2}}, \quad m<n .
$$

From Equation (4), Bohr predicted the following relationship.

$$
h v=-E_{m}+E_{n} \text {. }
$$

The energy of the hydrogen atom is discontinuous. Bohr thought that when the electron transitions from a state with energy $E_{n}$ to a state with energy $E_{m}$, the electron emits a photon with energy $h v$. He also obtained the following formula for energy levels. 


$$
E_{n}=-\frac{h c R}{n^{2}} .
$$

At the time, the value of $c R$ was known through experiment, but details concerning $R$ were not known. Thus Bohr decided to derive the energy levels of the hydrogen atom using another method.

First, Bohr considered the case where the electron moves at constant speed around the atomic nucleus (proton). If $r$ is taken to be the radius of a circular orbit, and $v$ is taken to be the speed of the electron, then the following Newtonian equation of motion holds.

$$
\frac{m_{\mathrm{e}} v^{2}}{r}=\frac{1}{4 \pi \varepsilon_{0}} \frac{e^{2}}{r^{2}} .
$$

This equation indicates the equality of the centrifugal force acting on the electron (left side) and the Coulomb attraction received by the electron from the atomic nucleus. Here, the electron mass was set to $m_{\mathrm{e}}$, and the charge was set to $-e$.

Also, since the energy of the electron can be expressed by the sum of the kinetic energy $K$ and potential energy $V(r)$,

$$
E=\frac{m_{\mathrm{e}} v^{2}}{2}-\frac{1}{4 \pi \varepsilon_{0}} \frac{e^{2}}{r} .
$$

According to the Virial theorem, $2 K=-V(r)$ in the case of a circular orbit, and thus the energy can be written as follows. (The discussion here concerns a circular orbit as a special form of an elliptical orbit.)

$$
\begin{gathered}
E=-\frac{m_{\mathrm{e}} v^{2}}{2} . \\
E=-\frac{1}{2} \frac{1}{4 \pi \varepsilon_{0}} \frac{e^{2}}{r} .
\end{gathered}
$$

Here, if both sides of Equation (10) are squared,

$$
E^{2}=\frac{1}{4}\left(\frac{1}{4 \pi \varepsilon_{0}}\right)^{2} \frac{e^{4}}{r^{2}}
$$

Next, the following equation is obtained by dividing Equations (11) by Equation (9).

$$
E=-\frac{m_{\mathrm{e}}}{2}\left(\frac{1}{4 \pi \varepsilon_{0}}\right)^{2} \frac{e^{4}}{\left(m_{\mathrm{e}} v r\right)^{2}} .
$$

Incidentally, the angular momentum $L$ when an electron moves in a circle can be expressed as $m v r$. Here, if the number $n$ is affixed to the energy $E$ and angular momentum $L$, then Equation (12) becomes as follows.

$$
E_{n}=-\frac{m_{\mathrm{e}}}{2}\left(\frac{e^{2}}{4 \pi \varepsilon_{0}}\right)^{2} \frac{1}{L_{n}^{2}} .
$$

The energy in Equation (6) could be found through calculation. Bohr believed Equations (6) and (13) to be equal. Thus,

$$
\frac{h c R}{n^{2}}=\frac{m_{\mathrm{e}}}{2}\left(\frac{e^{2}}{4 \pi \varepsilon_{0}}\right)^{2} \frac{1}{L_{n}^{2}} .
$$

It was found that $L$, for which a unique value was not known, could be expressed with the following equation.

$$
L_{n}=\frac{e^{2}}{4 \pi \varepsilon_{0}}\left(\frac{m_{\mathrm{e}}}{2 h c R}\right)^{1 / 2} \cdot n
$$


Bohr substituted in the not very precise numeric values for physical quantities that were known at the time and conjectured $L_{n}$ to be as follows.

$$
L_{n}=\frac{h}{2 \pi} n=n \hbar, \quad n=1,2, \cdots
$$

If Equation (16) is assumed, then Equation (13) becomes as follows.

$$
\begin{gathered}
E_{n}=-\frac{m_{\mathrm{e}}}{2}\left(\frac{e^{2}}{4 \pi \varepsilon_{0}}\right)^{2} \frac{1}{\hbar^{2} n^{2}} \\
=-\frac{m_{\mathrm{e}} c^{2}}{2}\left(\frac{e^{2}}{4 \pi \varepsilon_{0} \hbar c}\right)^{2} \frac{1}{n^{2}} \\
=-\frac{\alpha^{2} m_{\mathrm{e}} c^{2}}{2 n^{2}} .
\end{gathered}
$$

This made it possible to find the energy levels of the hydrogen atom. Here, $\alpha$ is the following fine-structure constant.

$$
\alpha=\frac{e^{2}}{4 \pi \varepsilon_{0} \hbar c} .
$$

Equation (17) is not a logically derived equation. It is an equation derived by assuming the quantum condition in Equation (16).

At the time, L. de. Broglie noticed that light, thought to be a wave in the classical theory, exhibits particle characteristics. He also predicted that the electron, thought to be a particle, would exhibit wave characteristics. He also assumed that when the wavelength $\lambda$ of the wave accompanying an electron in circular motion satisfies the following relationship, that electron is in a stable state.

$$
\frac{2 \pi r}{\lambda}=n, \quad n=1,2, \cdots
$$

The following relationship holds between the momentum $p$ and wavelength $\lambda$ of the electron.

$$
\lambda=\frac{h}{p} .
$$

Substituting the $\lambda$ of Equation (20) into Equation (19) and also taking into account Equation (16), the following equation can be derived.

$$
2 \pi r p_{n}=2 \pi L_{n}=n h, \quad n=1,2, \cdots
$$

According to de Broglie, Bohr's quantum condition was able to acquire a substantive meaning, and thus it came to be that the energy levels of the hydrogen atom in Equation (17), found by assuming Equation (16), were believed to be correct.

Also, if $E_{n}$ in Equation (17) is substituted into Equation (10), then the following formula can be derived as the orbital radius of the electron.

$$
r_{n}=4 \pi \varepsilon_{0} \frac{\hbar^{2}}{m_{\mathrm{e}} \mathrm{e}^{2}} n^{2}, \quad n=1,2, \cdots
$$

\section{Discussion of Bohr's Quantum Condition}

\subsection{Relationship Enfolded in Bohr's Quantum Condition}

Bohr thought the following quantum condition was necessary to find the energy levels of the hydrogen atom.

$$
m_{\mathrm{e}} v_{n} \cdot 2 \pi r_{n}=2 \pi n \hbar
$$


In Bohr's theory, the energy of the hydrogen atom is treated non-relativistically, and thus here the momentum of the electron is taken to be $m_{\mathrm{e}} v$. Also, the Planck constant $h$ can be written as follows (Suto, 2015):

$$
\hbar=\frac{h}{2 \pi}=\frac{m_{\mathrm{e}} c \lambda_{\mathrm{C}}}{2 \pi} .
$$

$\lambda_{\mathrm{C}}$ is the Compton wavelength of the electron.

When Equation (24) is used, the fine-structure constant $\alpha$ can be expressed as follows.

$$
\alpha=\frac{e^{2}}{4 \pi \varepsilon_{0} \hbar c}=\frac{e^{2}}{2 \varepsilon_{0} m_{\mathrm{e}} c^{2} \lambda_{\mathrm{C}}} .
$$

Also, the classical electron radius $r_{\mathrm{e}}$ is defined as follows.

$$
r_{\mathrm{e}}=\frac{e^{2}}{4 \pi \varepsilon_{0} m_{\mathrm{e}} c^{2}} .
$$

If $r_{\mathrm{e}} / \alpha$ is calculated here,

$$
\frac{r_{\mathrm{e}}}{\alpha}=\frac{\lambda_{\mathrm{C}}}{2 \pi} .
$$

If Equation (22) is written using $r_{\mathrm{e}}$ and $\alpha$, the result is as follows.

$$
r_{n}=4 \pi \varepsilon_{0} \frac{\hbar^{2}}{m_{\mathrm{e}} e^{2}} n^{2}=\frac{e^{2}}{4 \pi \varepsilon_{0} m_{\mathrm{e}} c^{2}}\left(\frac{4 \pi \varepsilon_{0} \hbar c}{e^{2}}\right)^{2} n^{2}=\frac{r_{\mathrm{e}}}{\alpha^{2}} n^{2} .
$$

Next, if $\hbar$ in Equation (24) and $r_{n}$ in Equation (28) are substituted into Equation (23),

$$
m_{\mathrm{e}} v_{n} \cdot 2 \pi \frac{r_{\mathrm{e}}}{\alpha^{2}} n^{2}=2 \pi n \frac{m_{\mathrm{e}} c \lambda_{\mathrm{C}}}{2 \pi} .
$$

If Equation (27) is also used, then Equation (29) can be written as follows.

$$
m_{\mathrm{e}} v_{n} \cdot 2 \pi \frac{r_{\mathrm{e}}}{\alpha^{2}} n^{2}=2 \pi n \frac{m_{\mathrm{e}} c r_{\mathrm{e}}}{\alpha} .
$$

From this, the following relationship can be derived.

$$
\frac{v_{n}}{c}=\frac{\alpha}{n}
$$

\subsection{Various Formulas Derivable from Equation (31)}

Bohr's quantum condition:

It was possible to derive Equation (31) from Bohr's quantum condition (23), and thus it should be possible to derive Equation (23) from Equation (31). First, both sides of Equation (31) are multiplied by $m_{\mathrm{e}} \cdot 2 \pi r_{n}$. Next, when the value of Equation (22) is substituted for $r_{n}$ on the right side,

$$
m_{\mathrm{e}} v_{n} \cdot 2 \pi r_{n}=\frac{m_{\mathrm{e}} c}{n}\left(\frac{e^{2}}{4 \pi \varepsilon_{0} \hbar c}\right) 2 \pi \cdot 4 \pi \varepsilon_{0} \frac{\hbar^{2}}{m_{\mathrm{e}} e^{2}} n^{2}=2 \pi n \hbar .
$$

With this, it was possible to derive Bohr's quantum condition (23) from Equation (31).

Bohr's energy levels (17):

When both sides of Equation (31) are squared, and then multiplied by $m_{\mathrm{c}} / 2$,

$$
\frac{1}{2} \frac{m_{\mathrm{e}} v_{n}^{2}}{c^{2}}=\frac{1}{2} \frac{m_{\mathrm{e}} \alpha^{2}}{n^{2}}
$$

Hence,

$$
E_{n}=-\frac{1}{2} m_{\mathrm{e}} v_{n}^{2}=-\frac{\alpha^{2} m_{\mathrm{e}} c^{2}}{2 n^{2}}
$$


If Equation (31) is taken as a departure point, the energy levels of the hydrogen atom derived by Bohr can be derived immediately. Equation (31) has tremendous power. However, from a relativistic perspective, $(1 / 2) m_{\mathrm{e}} v_{n}^{2}$ is an approximation of the kinetic energy of the electron. Therefore, the energy in Equation (34) is also an approximation of the true value.

\subsection{Calculation of the Left Side of Equation (23)}

In this paper, Bohr's quantum condition is treated relativistically. Therefore, if $m_{\mathrm{e}} v_{n}$ in Equation (23) is replaced with $p_{n}$, the quantum condition becomes as follows.

$$
p_{n} \cdot 2 \pi r_{n}=2 \pi n \hbar .
$$

Now, in Equation (23), it is assumed that the left side and right side are equal. However, in this paper, the right side is determined by actually calculating the left side of Equation (35).

One of the most important relationships in the Special Theory of Relativity (STR) is as follows:

$$
\left(m_{0} c^{2}\right)^{2}+\boldsymbol{p}^{2} c^{2}=\left(m c^{2}\right)^{2} .
$$

Here, $m c^{2}$ is the relativistic energy of an object or a particle, and $m_{0} c^{2}$ is the rest mass energy.

Currently, Einstein's relationship (36) is used to describe the energy and momentum of particles in free space, but for explaining the behavior of bound electrons inside atoms, opinion has shifted to quantum mechanics as represented by equations such as the Dirac's relativistic wave equation.

For reasons such as these, there was no search for a relationship between energy and momentum applicable to an electron in the hydrogen atom. However, the author has ventured to take up this problem, and derived the following relationship (Suto, 2011; Suto, 2018a):

$$
E_{\mathrm{re}, n}^{2}+\boldsymbol{p}_{n}^{2} c^{2}=\left(m_{\mathrm{e}} c^{2}\right)^{2}, \quad n=1,2, \cdots
$$

However,

$$
E_{\mathrm{re}, n}=m_{\mathrm{e}} c^{2}+E_{n}=m_{\mathrm{e}} c^{2}-K_{n}==m_{\mathrm{e}} c^{2}+\frac{1}{2} V\left(r_{n}\right) .
$$

Here, $E_{\mathrm{re}, n}$ is the relativistic energy of the electron, described with an absolute scale.

The following formula can be derived from Equation (37). (See Appendix A)

$$
E_{\mathrm{re}, n}=m_{\mathrm{e}} c^{2}\left(1+\frac{v_{n}^{2}}{c^{2}}\right)^{-1 / 2} \text {. }
$$

However, Equation (39) is insufficient as a formula describing the micro world. The velocity of the electron is included in the equation, and the discrete energy levels characteristic of quantum mechanics have not been incorporated.

Thus, to solve this problem, Equation (39) is rewritten as follows using Equation (31).

$$
E_{\mathrm{r}, n}=m_{\mathrm{e}} c^{2}\left(1+\frac{\alpha^{2}}{n^{2}}\right)^{-1 / 2} \text {. }
$$

Also, $E_{\mathrm{re}, n}$ defined with Equation (38) can be defined as follows.

$$
E_{\mathrm{re}, n}=m_{n} c^{2}
$$

From this,

$$
m_{n}=m_{\mathrm{e}}\left(1+\frac{\alpha^{2}}{n^{2}}\right)^{-1 / 2} .
$$

Also, Equation (40) can be written as follows. 


$$
E_{\mathrm{re}, n}=m_{\mathrm{e}} c^{2}\left(\frac{n^{2}}{n^{2}+\alpha^{2}}\right)^{1 / 2} .
$$

When $E_{\mathrm{r}, n}$ is determined, the $p_{n}$ in Equation (37) is also determined as follows.

$$
p_{n}=m_{\mathrm{e}} c\left(\frac{\alpha^{2}}{n^{2}+\alpha^{2}}\right)^{1 / 2} \text {. }
$$

Incidentally, $E_{n}$ in Equation (10) can be written as follows.

$$
E_{n}=-\frac{1}{2} \frac{1}{4 \pi \varepsilon_{0}} \frac{e^{2}}{r_{n}} .
$$

Also, if Equation (43) is taken into account, the $E_{n}$ in Equation (38) can be written as follows.

$$
E_{n}=-K_{n}=E_{\mathrm{re}, n}-m_{\mathrm{e}} c^{2}=m_{\mathrm{e}} c^{2}\left[\left(\frac{n^{2}}{n^{2}+\alpha^{2}}\right)^{1 / 2}-1\right]
$$

When this $E_{n}$ is substituted into Equation (45) and the equation is rearranged, the following $r_{n}$ is found.

$$
r_{n}=\frac{r_{\mathrm{e}}}{2}\left(1+\frac{n^{2}}{\alpha^{2}}\right)\left[1+\left(1+\frac{\alpha^{2}}{n^{2}}\right)^{-1 / 2}\right] .
$$

Now the $p_{n}$ and $r_{n}$ substituted into the left side of Equation (35) are determined (i.e., Equations (44) and (47)).

If these values are substituted into the left side of Equation (35), the result is as follows.

$$
m_{\mathrm{e}} c\left(\frac{\alpha^{2}}{n^{2}+\alpha^{2}}\right)^{1 / 2} \cdot 2 \pi \frac{r_{\mathrm{e}}}{2}\left(\frac{n^{2}+\alpha^{2}}{\alpha^{2}}\right)\left[1+\left(\frac{n^{2}}{n^{2}+\alpha^{2}}\right)^{1 / 2}\right]=m_{\mathrm{e}} c \cdot 2 \pi \frac{r_{\mathrm{e}}}{2} \frac{n}{\alpha}\left[1+\left(1+\frac{\alpha^{2}}{n^{2}}\right)^{1 / 2}\right] .
$$

If Equations (27) and (24) are used here, then Equation (48) becomes,

$$
m_{\mathrm{e}} c \cdot 2 \pi \frac{r_{\mathrm{e}}}{2} \frac{n}{\alpha}\left[1+\left(1+\frac{\alpha^{2}}{n^{2}}\right)^{1 / 2}\right]=2 \pi n \hbar \cdot \frac{1}{2}\left[1+\left(1+\frac{\alpha^{2}}{n^{2}}\right)^{1 / 2}\right] .
$$

Hence,

$$
p_{n} \cdot 2 \pi r_{n}=2 \pi n \hbar \cdot \frac{1}{2}\left[1+\left(1+\frac{\alpha^{2}}{n^{2}}\right)^{1 / 2}\right]
$$

Next, if the right side of Equation (50) is expanded, the result is as follows.

$$
2 \pi n \hbar \cdot \frac{1}{2}\left[1+\left(1+\frac{\alpha^{2}}{n^{2}}\right)^{1 / 2}\right] \approx 2 \pi n \hbar \cdot \frac{1}{2}\left[1+\left(1+\frac{\alpha^{2}}{2 n^{2}}-\frac{\alpha^{4}}{8 n^{4}}+\frac{\alpha^{6}}{16 n^{6}}\right)\right] .
$$

This yields,

$$
p_{n} \cdot 2 \pi r_{n} \approx 2 \pi n \hbar\left(1+\frac{\alpha^{2}}{4 n^{2}}-\frac{\alpha^{4}}{16 n^{4}}+\frac{\alpha^{6}}{32 n^{6}}\right) .
$$

As $n$ increases, Equation (52) converges to Equation (35). Due to the above discussion,

$$
p_{n} \cdot 2 \pi r_{n} \neq 2 \pi n \hbar \text {. }
$$

Considered from the perspective of the theory of relativity, Bohr's quantum condition (35) does not strictly hold. Also, if the relationship of Equation (42) is used, Equation (50) can be written as follows. (See Appendix B) 


$$
p_{n} \cdot 2 \pi r_{n}=2 \pi n \hbar \cdot \frac{1}{2}\left(1+\frac{m_{\mathrm{e}}}{m_{n}}\right)
$$

Incidentally, there is the following quantum condition of Sommerfeld, as an extension of Bohr's quantum condition to a 3-dimensional elliptical orbit.

$$
\oint p d s=2 \pi n \hbar
$$

However, if Equation (53) is taken into account, Equation (55) does not strictly hold.

\section{Derivation of a Previously Unknown Equation for Kinetic Energy}

In classical mechanics, the kinetic energy of the electron is defined as follows.

$$
K=\frac{1}{2} m_{\mathrm{e}} v^{2}
$$

However, the mass of a moving electron varies (in the STR, it is predicted that the mass of a moving object will increase, but the mass of an electron moving within an atom will decrease). Therefore, Equation (56) does not strictly hold. Now, can the kinetic energy of an electron in a hydrogen atom be described with the following formula?

$$
K=\frac{1}{2} m_{n} v_{n}^{2}
$$

To address this problem, let us first discuss the momentum of the electron.

\subsection{Momentum of the Electron in a Hydrogen Atom}

The momentum of the electron $p_{n}$ is given by Equation (44), but does the following formula hold?

$$
p_{n}=m_{n} v_{n} \text {. }
$$

If the values of $m_{n}$ in Equation (42) and $v_{n}$ in Equation (31) are substituted into the right side of Equation (58),

$$
m_{n} v_{n}=m_{\mathrm{e}}\left(1+\frac{\alpha^{2}}{n^{2}}\right)^{-1 / 2} \cdot \frac{\alpha c}{n}=m_{\mathrm{e}} c\left(\frac{\alpha^{2}}{n^{2}+\alpha^{2}}\right)^{1 / 2}=p_{n}
$$

From this, it is evident that Equation (58) is correct.

\subsection{Kinetic Energy of the Electron in a Hydrogen Atom}

Does the following formula for kinetic energy used in quantum mechanics hold?

$$
K_{n}=\frac{p_{n}^{2}}{2 m_{\mathrm{e}}} .
$$

Equation (42) can be written as follows.

$$
\left(\frac{n^{2}}{n^{2}+\alpha^{2}}\right)^{1 / 2}=\frac{m_{n}}{m_{\mathrm{e}}}
$$

Also, the formula for momentum of the electron Equation (44) can be written as follows.

$$
p_{n}=m_{\mathrm{e}} c\left(1-\frac{n^{2}}{n^{2}+\alpha^{2}}\right)^{1 / 2}=m_{\mathrm{e}} c\left(1-\frac{m_{n}^{2}}{m_{\mathrm{e}}^{2}}\right)^{1 / 2} .
$$

From this, $p_{n}$ can be defined as follows.

$$
p_{n}=c\left(m_{\mathrm{e}}^{2}-m_{n}^{2}\right)^{1 / 2}
$$

When Equations (58) and (63) are combined, 


$$
p_{n}=m_{n} v_{n}=c\left(m_{\mathrm{e}}^{2}-m_{n}^{2}\right)^{1 / 2} .
$$

If Equation (31) is taken into account, the following relationship can also be derived from Equation (64).

$$
\frac{v_{n}}{c}=\frac{\left(m_{\mathrm{e}}^{2}-m_{n}^{2}\right)^{1 / 2}}{m_{n}}=\frac{\alpha}{n} .
$$

From this, it is evident that there is the following relationship between the fine-structure constant and the mass of the electron.

$$
\alpha=\frac{\left(m_{\mathrm{e}}^{2}-m_{1}^{2}\right)^{1 / 2}}{m_{1}} .
$$

Incidentally, it is also clear from Equations (38) and (41) that the kinetic energy of the electron $K_{n}$ can be defined as follows.

$$
K_{n}=\left(m_{\mathrm{e}}-m_{n}\right) c^{2}
$$

However, in this paper the aim is to find a formula for kinetic energy similar to Equations (56) and (57).

Now, let us write Equation (60) as follows, and determine the value of A.

$$
K_{n}=\frac{p_{n}^{2}}{\mathrm{~A}} .
$$

When Equations (63) and (67) are used,

$$
\mathrm{A}=\frac{p_{n}^{2}}{K_{n}}=\frac{\left(m_{\mathrm{e}}-m_{n}\right)\left(m_{\mathrm{e}}+m_{n}\right) c^{2}}{\left(m_{\mathrm{e}}-m_{n}\right) c^{2}}=m_{\mathrm{e}}+m_{n} .
$$

From this, the formula for the kinetic energy of an electron in the hydrogen atom is not Equation (60), and becomes as follows.

$$
K_{n}=\frac{p_{n}^{2}}{m_{\mathrm{e}}+m_{n}} .
$$

Here, if Equation (70) is written to imitate the form of Equation (57), the result is as follows.

$$
K_{n}=\frac{m_{n} v_{n}^{2}}{1+m_{\mathrm{e}} / m_{n}} .
$$

Equations (56) and (57) are approximations of Equation (71). However, the velocity of the electron in the atom is not a basic physical quantity. Therefore, Equation (71) is inferior to Equation (70).

\section{Discussion}

4.1 Importance of Equations (50) and (54)

The author has derived the following relationship in another paper (Suto, 2017d):

$$
\left(1+\frac{\alpha^{2}}{n^{2}}\right)^{1 / 2}=\frac{m_{\mathrm{e}}}{m_{n}}=\frac{r_{n}}{r_{n}-r_{\mathrm{e}} / 2}
$$

If 1 is added to each side of Equation (72), it becomes as follows.

$$
1+\left(1+\frac{\alpha^{2}}{n^{2}}\right)^{1 / 2}=\frac{m_{\mathrm{e}}+m_{n}}{m_{n}}=\frac{2 r_{n}-r_{\mathrm{e}} / 2}{r_{n}-r_{\mathrm{e}} / 2} .
$$

When this relationship is taken into account, Equation (50) can be written as follows. 


$$
p_{n} \cdot 2 \pi r_{n}=2 \pi n \hbar\left(\frac{r_{n}-r_{\mathrm{e}} / 4}{r_{n}-r_{\mathrm{e}} / 2}\right) .
$$

Here, $r_{\mathrm{e}} / 4$ is thought to be the radius of the proton (Suto, 2017b):

Also, $r=r_{\mathrm{e}} / 2$ is the orbital radius when the relativistic energy of the electron becomes zero (Suto, 2014): However, it is unclear whether these formulas (Equations (50), (54), and (74)) that are more precise than Bohr's quantum condition have a deep physical significance.

Incidentally, if the values of $p_{n}$ and $r_{n}$ derived in this paper are substituted into the left side of Equation (35), then the right side is determined. However, Equations (50), (54) and the like cannot be derived if Equation (31) is not assumed. Therefore, it is not desirable to assume these formulas from the beginning instead of Equation (35).

For Equations (50), (54) and the like, it should not be assumed that the right and left sides are the same; the interpretation should be that when the left side is calculated, it becomes the right side. In this paper, it is thought that Equation (31) is more substantial than Bohr's quantum condition as a quantum condition.

\subsection{Kinetic Energy of the Electron (Comparison of This Paper and Classical Quantum Theory)}

4.2.1 A. Sommerfeld defined kinetic energy as the energy a moving object has in excess of the stationary object. Therefore, if the rest mass of the object is $m_{0}$ and the mass when moving is $m$, the kinetic energy of the object can be described by the following formula (Sommerfeld, 1923):

$$
K_{\mathrm{SO}}=c^{2}\left(m-m_{0}\right)=m_{0} c^{2}\left[\frac{1}{\left(1-\beta^{2}\right)^{1 / 2}}-1\right], \quad \beta=\frac{v}{c} .
$$

Here, the relativistic energy of a particle $E_{\mathrm{re}}$ is $m c^{2}$, and thus the following relationship holds.

$$
E_{\mathrm{re}}>m_{0} c^{2} \text {. }
$$

Sommerfeld believed that Equation (75), which can be derived from Equation (36), can also be applied to the electron in a hydrogen atom. If, following Equation (75), the rest mass of the electron is taken to be $m_{\mathrm{e}}$, then the kinetic energy of the electron $K_{\text {so }}$ can be described with the following two equations. (Here, $K_{\text {so }}$ refers to the kinetic energy predicted by Sommerfeld.)

$$
\begin{gathered}
K_{\mathrm{SO}}=c^{2}\left(m-m_{\mathrm{e}}\right)=m_{\mathrm{e}} c^{2}\left[\frac{1}{\left(1-\beta^{2}\right)^{1 / 2}}-1\right] . \\
K_{\mathrm{SO}}=E_{\mathrm{re}}-m_{\mathrm{e}} c^{2} .
\end{gathered}
$$

Incidentally, the energy of the hydrogen atom derived by Bohr becomes a negative value. However, Sommerfeld did not take that to be a problem. This is because the energy becomes positive if the rest mass energy is added to the energy of the hydrogen atom, and the energy is described relativistically. That is,

$$
E_{\mathrm{re}}=E+m_{\mathrm{e}} c^{2}, \quad 0<E_{\mathrm{re}}<m_{\mathrm{e}} c^{2} .
$$

However, between $E$ and $K$, there is the relationship $-E=K$. Therefore, Equation (79) can be written as follows.

$$
E_{\mathrm{re}}=m_{\mathrm{e}} c^{2}-K
$$

Thus, $K$ is

$$
K=m_{\mathrm{e}} c^{2}-E_{\mathrm{re}} .
$$

The relativistic energy $E_{\mathrm{re}}$ of an electron forming a hydrogen atom is smaller than the rest mass energy of the electron. Therefore, the formula for the kinetic energy of an electron in a hydrogen atom is not Equation (77). If the kinetic energy of the electron predicted by this paper is indicated $K_{\mathrm{SU}, n}$, then $K_{\mathrm{SU}, n}$ is given by the following formula. (Here, $K_{\mathrm{SU}}$ signifies the kinetic energy predicted by this paper (Suto).)

$$
K_{\mathrm{SU}, n}=m_{\mathrm{e}} c^{2}-m_{n} c^{2}=m_{\mathrm{e}} c^{2}\left[1-\left(1+\frac{\alpha^{2}}{n^{2}}\right)^{-1 / 2}\right] .
$$


In contrast, the formula of Sommerfeld Equation (75) can be written as follows by using Equation (31).

$$
K_{\mathrm{SO}, n}=m_{\mathrm{e}} c^{2}\left[\left(1-\frac{\alpha^{2}}{n^{2}}\right)^{-1 / 2}-1\right] .
$$

Now, if a Taylor expansion is performed on the right side of Equations (83) and (82),

$$
\begin{aligned}
& K_{\mathrm{SO}, n} \approx m_{\mathrm{e}} c^{2}\left[\left(1+\frac{\alpha^{2}}{2 n^{2}}+\frac{3 \alpha^{4}}{8 n^{4}}+\frac{5}{16} \frac{\alpha^{6}}{n^{6}}\right)-1\right] . \\
& K_{\mathrm{SU}, n} \approx m_{\mathrm{e}} c^{2}\left[1-\left(1-\frac{\alpha^{2}}{2 n^{2}}+\frac{3 \alpha^{4}}{8 n^{4}}-\frac{5 \alpha^{6}}{16 n^{6}}\right)\right] .
\end{aligned}
$$

Rewriting these formulas, the following is obtained.

$$
\begin{gathered}
K_{\mathrm{SO}, n} \approx \frac{\alpha^{2}}{2 n^{2}} m_{\mathrm{e}} c^{2}\left(1+\frac{3 \alpha^{2}}{4 n^{2}}+\frac{5}{8} \frac{\alpha^{4}}{n^{4}}\right) . \\
K_{\mathrm{SU}, n} \approx \frac{\alpha^{2}}{2 n^{2}} m_{\mathrm{e}} c^{2}\left(1-\frac{3 \alpha^{2}}{4 n^{2}}+\frac{5}{8} \frac{\alpha^{4}}{n^{4}}\right) .
\end{gathered}
$$

When $n=1$, Equations (83), (82) and (17) become as follows.

$$
\begin{gathered}
K_{\mathrm{SO}, n}=-E_{\mathrm{s}, n}=13.60515 \mathrm{eV} . \\
K_{\mathrm{SU}, n}=-E_{\mathrm{su}, n}=13.60624 \mathrm{eV} . \\
K_{\mathrm{BO}, n}=\frac{\alpha^{2} m_{\mathrm{e}} c^{2}}{2 n^{2}}=13.60569 \mathrm{eV} .
\end{gathered}
$$

Here, if the ratio of the two kinetic energies is taken,

$$
\frac{K_{\mathrm{SO}, n}}{K_{\mathrm{SU}, n}}=0.99992 .
$$

The difference between $K_{\mathrm{SO}, n}$ and $K_{\mathrm{SU}, n}$ is so small that it is difficult to discern by experiment. In conventional physics, if a theoretical value and measured value are regarded as the same, then the thinking has been that the correctness of the theory has been demonstrated. (That is, the Sommerfeld theory was also thought to be correct because the theoretical value and experimental value were regarded as matching.)

However, this paper has presented an example where the theory's correctness is not necessarily demonstrated, even though the theoretical value and measurement value are regarded as the same. Physics has entered a new era.

Incidentally, since Equation (37) could be derived, there is a considerable broadening of the energy region that can be handled. Whereas the region of energy that can be handled with Equation (37) $E_{\mathrm{re}}$ is $-m_{\mathrm{e}} c^{2}<E_{\mathrm{re}}<m_{\mathrm{e}} c^{2}$, the region that can be handled with Equation (17) is $-\alpha^{2} m_{\mathrm{e}} c^{2} / 2 n^{2}<E_{n}<0$. Taking the ratio of these two energy domains,

$$
\frac{2 m_{\mathrm{e}} c^{2}}{-E_{\mathrm{so}, 1}}=75,112.46
$$

It is very significant that Equation (37) could be derived (Suto, 2018b).

\subsubsection{Correction of the Hamiltonian}

The Hamiltonian used in quantum mechanics has the following form.

$$
H=m_{\mathrm{e}} c^{2}(\gamma-1)-\frac{1}{2} \frac{e^{2}}{r}, \quad \gamma=\left(1-\frac{v^{2}}{c^{2}}\right)^{-1 / 2} .
$$

However, according to this paper, the correct Hamiltonian takes the following form. 


$$
H=m_{\mathrm{e}} c^{2}\left(1-\gamma^{\prime}\right)-\frac{1}{2} \frac{e^{2}}{r}, \quad \gamma^{\prime}=\left(1+\frac{v^{2}}{c^{2}}\right)^{-1 / 2}=\left(1+\frac{\alpha^{2}}{n^{2}}\right)^{-1 / 2} .
$$

4.3 Physical Quantities Derived from Equation (31)

\subsubsection{Kinetic Energy of the Electron}

First, if both sides of Equation (31) are squared, and multiplied by $m_{n}^{2} /\left(m_{\mathrm{e}}+m_{n}\right)$,

$$
\frac{m_{n}^{2}}{m_{\mathrm{e}}+m_{n}} \cdot \frac{v_{n}^{2}}{c^{2}}=\frac{\alpha^{2}}{n^{2}} \cdot \frac{m_{n}^{2}}{m_{\mathrm{e}}+m_{n}} .
$$

From this, the kinetic energy of the electron $K_{n}$ is,

$$
K_{n}=\frac{m_{n}^{2} v_{n}^{2}}{m_{\mathrm{e}}+m_{n}}=\frac{\alpha^{2} c^{2}}{n^{2}} \cdot \frac{m_{n}^{2}}{m_{\mathrm{e}}+m_{n}} .
$$

If the relationship in Equation (61) is used here,

$$
K_{n}=\frac{\alpha^{2} c^{2}}{n^{2}}\left(\frac{n^{2}}{n^{2}+\alpha^{2}}\right) m_{\mathrm{e}}^{2} \cdot \frac{1}{m_{\mathrm{e}}\left[1+\left(\frac{n^{2}}{n^{2}+\alpha^{2}}\right)^{1 / 2}\right]} .
$$

Next, the following formula is multiplied with the numerator and denominator,

$$
1-\left(\frac{n^{2}}{n^{2}+\alpha^{2}}\right)^{1 / 2} \text {. }
$$

When this is done,

$$
\begin{gathered}
K_{n}=\frac{\alpha^{2} m_{\mathrm{e}} c^{2}}{n^{2}}\left(\frac{n^{2}}{n^{2}+\alpha^{2}}\right)\left[1-\left(\frac{n^{2}}{n^{2}+\alpha^{2}}\right)^{1 / 2}\right]\left(1-\frac{n^{2}}{n^{2}+\alpha^{2}}\right)^{-1} \\
=\frac{\alpha^{2} m_{\mathrm{e}} c^{2}}{n^{2}}\left(\frac{n^{2}}{n^{2}+\alpha^{2}}\right)\left[1-\left(\frac{n^{2}}{n^{2}+\alpha^{2}}\right)^{1 / 2}\right]\left(\frac{n^{2}+\alpha^{2}}{\alpha^{2}}\right) \\
=m_{\mathrm{e}} c^{2}\left[1-\left(\frac{n^{2}}{n^{2}+\alpha^{2}}\right)^{1 / 2}\right] .
\end{gathered}
$$

This enables derivation of Equation (82) from Equation (31).

4.3.2 Momentum of the Electron

If both sides of Equation (31) are multiplied by $m_{n}$,

$$
\frac{m_{n} v_{n}}{c}=\frac{\alpha}{n} m_{n}
$$

Next, if the relationship in Equation (61) is used, $p_{n}$ becomes as follows.

$$
p_{n}=m_{n} v_{n}=\frac{\alpha}{n} m_{\mathrm{e}} c\left(\frac{n^{2}}{n^{2}+\alpha^{2}}\right)^{1 / 2}=m_{\mathrm{e}} c\left(\frac{\alpha^{2}}{n^{2}+\alpha^{2}}\right)^{1 / 2} .
$$

This enables derivation of Equation (44) from Equation (31).

4.4 Energy of a Particle Moving in an Isolated System in Free Space

This is a digression from the theme of this paper, but let's try to find a formula for the kinetic energy of a particle from Equation (36). According to the STR, the relationship of mass $m$ and rest mass $m_{0}$ of a particle moving in free space is as follows. 


$$
m=\frac{m_{0}}{\left(1-\beta^{2}\right)^{1 / 2}} .
$$

Thus Equation (36) can be written as follows. (However, this is outside the atom, so $n$ is deleted.)

$$
m_{0}^{2} c^{4}\left(\frac{c^{2}}{c^{2}-v^{2}}\right)=m_{0}^{2} c^{4}+p^{2} c^{2} .
$$

If $p^{2}$ is found from this equation,

$$
p^{2}=m_{0}^{2} c^{2}\left(\frac{v^{2}}{c^{2}-v^{2}}\right)
$$

Also, the kinetic energy $K$ of the particle,

$$
K=m c^{2}-m_{0} c^{2}=m_{0} c^{2}\left[\left(\frac{c^{2}}{c^{2}-v^{2}}\right)^{1 / 2}-1\right] .
$$

$p$ and $K$ have been found, and thus $\mathrm{B}$ is found this time from the following equation.

$$
K=\frac{p^{2}}{\mathrm{~B}} \text {. }
$$

The details will be omitted, but when the calculation is carried out, B is as follows.

$$
\mathrm{B}=m_{0}\left[1+\frac{1}{\left(1-v^{2} / c^{2}\right)^{1 / 2}}\right]=m_{0}+m .
$$

Thus Equation (105) becomes as follows.

$$
K=\frac{p^{2}}{m_{0}+m} .
$$

This formula can also be written as follows.

$$
K=\frac{m v^{2}}{1+m_{0} / m} .
$$

In classical mechanics, mass does not depend on velocity, and thus $m_{0}=m$. Equation (107), the correct formula for kinetic energy in classical mechanics, was not used as the formula for kinetic energy, and the following well-known formula was used.

$$
K=\frac{1}{2} m_{0} v^{2}
$$

\section{Conclusion}

\subsection{Quantum Condition}

When the actually obtained physical quantities are substituted into the left side of Equation (35), the following values (right side) are obtained (however, $p_{n}$ in this case is not the non-relativistic momentum treated by Bohr; it is the relativistic momentum.)

$$
\begin{gathered}
p_{n} \cdot 2 \pi r_{n}=2 \pi n \hbar \cdot \frac{1}{2}\left[1+\left(1+\frac{\alpha^{2}}{n^{2}}\right)^{1 / 2}\right] . \\
p_{n} \cdot 2 \pi r_{n}=2 \pi n \hbar \cdot \frac{1}{2}\left(1+\frac{m_{\mathrm{e}}}{m_{n}}\right) .
\end{gathered}
$$




$$
p_{n} \cdot 2 \pi r_{n}=2 \pi n \hbar\left(\frac{r_{n}-r_{\mathrm{e}} / 4}{r_{n}-r_{\mathrm{e}} / 2}\right) .
$$

However, these formulas cannot be derived if Equation (113) is not assumed at the start. Therefore, it is undesirable to assume these formulas at the beginning as a quantum condition to replace Bohr's condition Equation (23). In this paper, the following quantum condition is proposed as a new quantum condition to replace Bohr's quantum condition.

$$
\frac{v_{n}}{c}=\frac{\alpha}{n}
$$

If non-relativistic physical expressions are written on the left side of Equation (113), then a non-relativistic approximation appears on the right side (Equations (32) and (34)). If, in contrast, relativistic physical expressions are written on the left side of Equation (113), then a relativistically accurate expression appears on the right side (Equations (98) and (100)).

\subsection{Formula for Kinetic Energy of the Electron}

The mass of the electron in a hydrogen atom depends on the velocity of the electron (as the velocity of the electron increases, the mass of the electron decreases.) Therefore, the kinetic energy of the electron cannot be explained with classical mechanics. That is,

$$
\begin{gathered}
K_{n} \neq \frac{1}{2} m_{\mathrm{e}} v_{n}^{2} . \\
K_{n} \neq \frac{1}{2} m_{n} v_{n}^{2} . \\
K_{n} \neq \frac{p_{n}^{2}}{2 m_{\mathrm{e}}} .
\end{gathered}
$$

Also, the kinetic energy of an electron in a hydrogen atom cannot be described even with the following formula of Sommerfeld which conforms to Einstein's energy-momentum relationship (36).

$$
K_{n} \neq m_{\mathrm{e}} c^{2}\left[\left(\frac{n^{2}}{n^{2}-\alpha^{2}}\right)^{1 / 2}-1\right]
$$

The kinetic energy of an electron in a hydrogen atom is defined as follows.

$$
K_{n}=\left(m_{\mathrm{e}}-m_{n}\right) c^{2}
$$

Also, the formulas for kinetic energy of an electron presented in this paper are as allows.

$$
\begin{gathered}
K_{n}=m_{\mathrm{e}} c^{2}\left[1-\left(\frac{n^{2}}{n^{2}+\alpha^{2}}\right)^{1 / 2}\right] . \\
K_{n}=\frac{p_{n}^{2}}{m_{\mathrm{e}}+m_{n}} . \\
K_{n}=\frac{m_{n} v_{n}^{2}}{1+m_{\mathrm{e}} / m_{n}} .
\end{gathered}
$$

Here, the velocity of the electron is included in Equation (121), and thus Equation (121) is a formula inferior to Equation (120).

However, the formula for the kinetic energy of an object moving in an isolated system in free space must include velocity. As a formula for the kinetic energy of an object moving in macro space, the following formulas are more accurate than Newton's formula. 


$$
\begin{gathered}
K=\frac{p^{2}}{m_{0}+m} . \\
K=\frac{m v^{2}}{1+m_{0} / m}=\frac{m_{0} v^{2}}{\left(1-v^{2} / c^{2}\right)^{1 / 2}\left\{1+\left(1-v^{2} / c^{2}\right)^{1 / 2}\right\}} .
\end{gathered}
$$

\subsection{Formula for the Momentum of the Electron in a Hydrogen Atom}

The momentum of the electron in a hydrogen atom is defined as follows.

$$
p_{n}=m_{n} v_{n} .
$$

The formula for momentum presented by this paper is as follows.

$$
\begin{gathered}
p_{n}=m_{\mathrm{e}} \mathrm{c}\left(\frac{\alpha^{2}}{n^{2}+\alpha^{2}}\right)^{1 / 2} . \\
p_{n}=c\left(m_{\mathrm{e}}^{2}-m_{n}^{2}\right)^{1 / 2} .
\end{gathered}
$$

\section{Acknowledgements}

In preparing the Introduction, the author referred to the writings in Japanese of Dr. H. Ezawa. Here I would like to express my gratitude to Dr. Ezawa. Also, I would like to express my thanks to the staff at ACN Translation Services for their translation assistance.

\section{Conflict of interests}

The authors declare that there is no conflict of interests regarding the publication of this paper.

\section{References}

Sommerfeld, A. (1923). Atomic Structure and Spectral Lines (p. 528). London: Methuen \& Co. Ltd.

Suto, K. (2011). An energy-momentum relationship for a bound electron inside a hydrogen atom. Physics Essays, 24(2), 301-307.

Suto, K. (2014). Previously unknown ultra-low energy level of the hydrogen atom whose existence can be predicted. Applied Physics Research, 6(6), 64-73.

Suto, K. (2015). An unknown physical constant missing from physics. Applied Physics Research, 7(5), 68-79.

Suto, K. (2017a). Elucidating the nature of the fine structure constant and indicating the existence of an unknown angular momentum. Applied Physics Research, 9(3), 17-22.

Suto, K. (2017b). Presentation of dark matter candidates. Applied Physics Research, 9(1), 70-76.

Suto, K. (2017c). Previously unknown physical formulas which hold in a hydrogen atom and are derived without using quantum mechanics. Applied Physics Research, 9(4), 7-16.

Suto, K. (2017d). Region of dark matter present in a hydrogen atom. Journal of Physical Mathematics, 8(4), 10.4172/2090-0902.1000252.

Suto, K. (2018a). Derivation of a relativistic wave equation more profound than Dirac's relativistic wave equation. Applied Physics Research, 10(6), 102-108.

Suto, K. (2018b). Potential energy of the electron in a hydrogen atom and a model of a virtual particle pair constituting the vacuum. Applied Physics Research, 10(4), 93-101.

\section{Appendix A}

In classical mechanics,

$$
m=\frac{p}{v} .
$$

And, in STR, 


$$
m=\frac{E}{c^{2}}
$$

If, further, we suppose that Equation (A2) describes a universal equivalence of energy and inertial mass, we can combine Equations (A1) and (A2) into a single statement:

$$
E=\frac{p c^{2}}{v} .
$$

Incidentally, in this paper Equation (A3) can be written as follows.

$$
p_{n} c=\frac{E_{\mathrm{re}, n} v_{n}}{c} .
$$

Here, if we substitute $p_{n} c$ in Equation (A4) into Equation (37) and rearrange, then the following value is obtained.

$$
E_{\mathrm{re}, n}=m_{\mathrm{e}} c^{2}\left(1+\frac{v_{n}^{2}}{c^{2}}\right)^{-1 / 2}
$$

\section{Appendix B}

Until Bohr introduced the Planck constant $h$, the only universal constants were $m, e, c$, and $\varepsilon$, and with this alone it was impossible to create a quantity with the dimension of length. Now, is the Planck constant really a universal constant on a par with $e$ and $c$ ?

This problem has already been discussed in another paper (Suto, 2017c):

According to the STR, the electron mass energy $E_{0}$ is given by the following formula.

$$
E_{0}=m_{\mathrm{e}} c^{2} \text {. }
$$

According to the law of conservation of momentum, a single photon with this energy is never emitted from an electron. However, if there is a photon with this energy in the natural world, the energy of this photon can be decomposed as follows.

$$
E_{0}=m_{\mathrm{e}} c \lambda_{\mathrm{C}} v_{\mathrm{C}}
$$

Here, $\lambda_{\mathrm{C}}$ is the following electron Compton wavelength.

$$
\lambda_{\mathrm{C}}=\frac{h}{m_{\mathrm{e}} c} .
$$

If the name "Compton frequency" is tentatively assigned to the quantity $v_{\mathrm{C}}$ that has newly appeared here, then the speed of light can be written as follows.

$$
c=\lambda_{\mathrm{C}} v_{\mathrm{C}}
$$

If $m_{\mathrm{e}} c \lambda_{\mathrm{C}}$ in Equation (B2) is written as $h$ to simplify, then Equation (B2) can be written as follows.

$$
E_{0}=h v_{\mathrm{C}} \text {. }
$$

Now, consider a case where an electron stationary in free space is attracted to a proton, and forms a hydrogen atom. The kinetic energy $K_{n}$ which the electron acquires at this time can be expressed as follows.

$$
\begin{gathered}
K_{n}=m_{\mathrm{e}} c^{2}-m_{n} c^{2} . \\
K_{n}=m_{\mathrm{e}} c^{2}-m_{\mathrm{e}} c^{2}\left(1+\frac{\alpha^{2}}{n^{2}}\right)^{-1 / 2} .
\end{gathered}
$$

If the ratio of $K_{n}$ and $E_{0}$ is taken here,

$$
\frac{K_{n}}{E_{0}}=1-\left(\frac{n^{2}}{n^{2}+\alpha^{2}}\right)^{1 / 2} .
$$

Taking into account the relationship in Equation (B2), 


$$
\frac{K_{n}}{m_{\mathrm{e}} c \lambda_{\mathrm{C}}}=\left[1-\left(\frac{n^{2}}{n^{2}+\alpha^{2}}\right)^{1 / 2}\right] \nu_{\mathrm{C}} .
$$

Here a frequency $v_{n}$ like the following is defined.

$$
\left[1-\left(\frac{n^{2}}{n^{2}+\alpha^{2}}\right)^{1 / 2}\right] v_{\mathrm{C}}=v_{n} .
$$

When this is done,

$$
K_{n}=h v_{n} .
$$

However, the kinetic energy acquired by the electron and the photonic energy $E_{n}$ emitted by the electron are equal, and thus Equation (B11) can also be written as follows.

$$
E_{n}=h v_{n} \text {. }
$$

Incidentally, if it is assumed that the Planck constant is nothing more than a symbol for combining $m_{\mathrm{e}} c \lambda_{\mathrm{C}}$, then it is desirable to write the fine- structure constant as follows.

$$
\alpha=\frac{e^{2}}{4 \pi \varepsilon_{0} \hbar c}=\frac{e^{2}}{2 \varepsilon_{0} \lambda_{\mathrm{C}}} \cdot \frac{1}{m_{\mathrm{e}} c^{2}} .
$$

The Planck constant is thought to be the minimum unit of angular momentum existing in the natural world, but that is not actually the case. The minimum unit of angular momentum exists separately (Suto, 2017a).

\section{Copyrights}

Copyright for this article is retained by the author(s), with first publication rights granted to the journal.

This is an open-access article distributed under the terms and conditions of the Creative Commons Attribution license (http://creativecommons.org/licenses/by/4.0/). 